\title{
Todo tiene su tiempo: trabajo agrícola y trabajo informal urbano en El Salvador
}

\author{
Douglas Marcouiller**
}

"Antes, éramos dueños de nuestros esclavos. Ahora sólo los alquilamos".

Edward R. Murrow, Harvest of Shame

"Según los algodoneros, las cortas se encuentran atrasadas en por lo menos 30 dias, debido a lo cual es urgente la presencia de unos quince mil cortadores".

El Diario de Hoy, 8 de enero de 1991.

Los economistas suelen modelar el movimiento de población rural hacia el sector informal urbano, movimiento a partir del cual los campesinos se vuelven carpinteros, mecánicos y fontaneros. Datos recientes en El Salvador, sin embargo, senalan precisamente el movimiento opuesto: trabajadores urbanos parecen estarse desplazando estacionalmente hacia tareas agricolas. Hasta ahora, la literatura sobre informalidad ha ignorado esta transformación de carpinteros en campesinos.

- Una versión de este trabajo fue presentada al XVI Congreso de Latin American Studies Association (LASA), celebrado en Washington D.C. en abril de 1991.

- Este pequeño trabajo ha sido leído por grandes críticos. El autor desea agradecer especialmente a Michael Conroy, Peter Gregory, Juan Pablo Porez Sainz y Michael Wise. Desafortunadamente, ninguno de ellos estuvo dispuesto a aceptar responsabilidades por los errores que aún quedan. 
De la misma forma en que la literatura sobre informalidad ha hecho caso omiso de la agricultura, los modelos de exportaciones agricolas han hecho caso omiso del mercado de trabajo urbano. De nuevo, la experiencia de El Salvador senala el problema. En un intento por relajar restricciones cambiarias, los paquetes neoliberales de ajuste generalmente promueven la producción y exportaciones agrícolas mediante la devaluación, reformas tributarias y arancelarias, subsidios a las exportaciones y crédito agrícola. Sin embargo, la oferta de trabajo agricola rural no es infinitamente elástica. Si se requiere trabajo agricola adicional de las ciudades, los mercados urbanos de trabajo condicionarán el éxito de tales políticas agrícolas.

Así, ya sea que se trate de analizar la informalidad urbana o la agricultura, se requiere de un modelo que integre a ambos. En este trabajo se ilustrará este punto para el caso salvadoreho. Primero, usando datos de la encuesta de hogares 1988-89, se describe un grupo de trabajadores del sector informal que está obteniendo ingresos insuficientes en trabajos inestables pero de fácil entrada. Luego se mostrará que el empleo agrícola estacional ha estado creciendo, y que la agricultura depende crecientemente de trabajadores de origen urbano. En una tercera parte, se integrarán estos dos elementos en un modelo de migración estacional en respuesta a variaciones en los ingresos esperados.

Todo ésto tiene implicaciones de política económica. El primero de junio de 1989, Alfredo Cristriani asumió la presidencia de El Salvador, con la promesa de que sólo los pobres serían privilegiados bajo el nuevo orden económico de ARENA.' Desde entonces, varios programas tendientes a enfrentar las necesidades de los pobres han sido puestos en marcha, entre ellos los proyectos comunales del Fondo de Inversión Social ${ }^{2}$. Estos programas compensatorios, sin embargo, buscan simplemente suavizar el golpe asestado a los pobres, cuyo verdadero "privllegio" es soportar la carga del ajuste estructural. Para descubrir el impacto de las políticas de ajuste sobre los pobres es necesario seguir la pista a través de todos los a veces inesperados senderos por los cuales estas políticas llevan a la economia. En este trabajo se senala uno de esos senderos.

\section{Trabajo Informal urbeno}

¿Está el trabajo en América Latina subempleado o sobreexplotado? ¿Son las disparidades en el ingreso generadas por la segmentación del mercado de trabajo, o es un mito el fracaso del mercado 3 ? A partir de la experiencia de los trabajadores urbanos, los analistas han desa- 
rrollado el concepto de "sector informal" urbano. Antes de considerar los resultados tabulados hasta ahora inéditos de una encuesta reciente sobre el sector en El Salvador, puede ser útil esbozar brevernente el fundamento teórico.

\section{A. Perspectlvas teóricas sobre el sector informal}

Hace casi 20 anos, una encuesta realizada en el área metropolitana de San Salvador encontró que el $30 \%$ de todas las familias vivian es extrema pobreza. Sorprendentemente, esas familias tenian tasas más altas de participación laboral que otras, y los trabajadores pobres trabajaban más horas que los no pobres. La causa de ingresos insuficientes, por lo tanto, no radicaba en el desempleo sino en el tipo de empleo que los pobres tenian'. Patrones como éste se encontraron en muchos paises en desarrollo, estimulando el interés por el no regulado mercado de trabajo del sector informal urbano. Pueden distinguirse tres perspectivas teóricas principales sobre el problema.

La primera perspectivas está asociada al trabajo del Instituto Penano para la Libertad y la Democracia, en particular al libro de Hernando de Soto, El Otro Senderó. La perspectiva se centra en la informalidad como actividad fuera de la ley. De acuerdo con esta perspectiva, las actividades fuera de la ley reflejan el intento de empresarios dinámicos de escapar la pesada regulación que les imponen los estados neomercantilistas de América Latina, que están supuestamente más preocupados por redistribuir la riqueza que por crearla? ${ }^{7}$. Como resultado, la actividad productiva se desplaza hacia unidades pequenas, que se encuentran irreguladas y poseen quizá un carácter subterráneo.

Una segunda perspectiva sobre la informalidad plantea el problema de manera diferente. En lugar de contrastar el sector informal con el formal, penetra a los dos sectores para integrar a los empleados en ambos, contrastándolos con su patronos ${ }^{\ominus}$. Esta perspectiva resalta la vivienda y los servicios informales como formas de pasar el grueso del costo de reproducción de la fuerza de trabajo a los mismos trabajadores, manteniendo bajos los salarios en las empresas formales.

La tercera visión está asociada con la PREALC, la oficina regional de la Organización Internacional del Trabajo. El enfoque de PREALC pone el énfasis en la generación insuficiente de empleo por parte de los sectores modernos en América Latina. Algunas veces se afirma que la "insuficiencia dinámica" del crecimiento refleja la insuficiencia de inversión en un sistema que transfiere el excedente económico hacla el exterior. Otras veces, el argumento se dirige hacia el uso de tecnologla intensiva en capital en la industria latinoamericana, tecnologla que no 
responde a la dotación local de factores sino a la composición de la producción bajo una distribución del ingreso altamente desigual, a políticas económicas inadecuadas y al alto costo de desarrollar tecnologías apropiadas ${ }^{9}$. Cualquiera que sea la causa, el excedente de fuerza de trabajo en las ciudades encuentra bloqueado su acceso al empleo en el sector moderno, por lo que tiene que encontrar su propio empleo. El excedente de trabajadores se "autogenera" empleo ${ }^{10}$. Victor Tokman ofrece un resumen conciso y autorizado de esta posición:

Incapaces de encontrar empleo en los sectores modemos y enfrentados a la necesidad de sobrevivir, los trabajadores excedentes tienen que encontrar actividades generadoras de ingreso que puedan desarrollar. Dada su falta de capital, tanto físico como humano, las actividades que estos trabajadores puedan desarrollar tienen que ser fácilmente accesibles. La organización de la producción es, por lo tanto, nudimentaria, sin ninguna división clara de la propiedad de los medios de producción y, como resultado de la competencia, el ingreso medio se convierte en la variable de ajuste".

Con frecuencia se hacen algunos refinamientos al argumento general de la PREALC: el sector informal es de suyo heterogéneo; puede ser el lugar de trabajo de trabajadores cesantes del sector formal, así como de nuevos migrantes que llegan a la ciudad; sirve para mantener salarios bajos a través de la competencia y para proveer bienes-salario baratos.

Para los fines de este ensayo, el punto central del modelo de la PREALC es su énfasis en las débiles barreras a la entrada a las actividades informales, que tienen lugar en empresas pequenas con escaso capital y en mercados competitivos. Los ingresos juegan el papel de "variable de ajuste" a medida que el empleo en el sector se expande ${ }^{12}$.

\section{B. Visión global del empleo Informal en el área urbana de El Sal- vador}

Las encuestas de hogares de El Salvador siguen a PREALC al distinguir entre los sectores moderno e informal. El sector informal estaría constituido por asalariados y familiares no remunerados que trabajan en establecimientos de menos de cinco trabajadores, así como empleados por cuenta propia y patronos que trabajan fuera de los campos de servicios profesionales, técnicos y administrativos ${ }^{13}$. La encuesta de la que se obtuvieron los datos que a continuación se presentan fue realizada entre octubre de 1988 y febrero de 1989. 
La encuesta muestra que la población económicamente activa urbana de El Salvador está dividida casi uniformemente entre los dos sectores (informal y modemo), que incluyen todas las ramas de la actividad económica (Cuadro 1). En la agricultura, la industria, la construcción y el transporte y las comunicaciones, el empleo se divide aproximadamente en partes iguales entre los sectores informal y moderno. En los establecimientos financieros, el empleo recae en su mayor parte sobre el sector moderno. El sector informal tiene un peso relativamente grande en el comercio, que es también la actividad en la que el empleo por cuenta propia es más común; $53 \%$ del total de empleados por cuenta propia se encuentran en el comercio y $55 \%$ de los empleados en el comercio son trabajadores por cuenta propia ${ }^{14}$.

\section{Cuadro 1}

DIstrlbuclón de la PEA urbana: Sector y Rama de actlvidad (miles de trabajadores)

\begin{tabular}{|l|r|r|r|c|}
\hline Rama & Total & Moderno & Informal & \% Informal \\
\hline Agricultura & 84.6 & 42.1 & 42.5 & 50.2 \\
Mineria & 11.6 & 8.1 & 3.5 & 29.8 \\
Industria & 167.1 & 88.2 & 78.9 & 47.2 \\
Elect/Gas/Agua & 7.9 & 6.6 & 1.3 & 16.6 \\
Construcción & 55.1 & 28.6 & 26.5 & 48.0 \\
Comercio & 199.0 & 44.0 & 155.0 & 77.9 \\
Transp/Comun. & 46.2 & 20.0 & 26.2 & 56.7 \\
Financiero & 25.8 & 22.0 & 2.8 & 10.9 \\
Servicios* & 188.5 & 140.4 & 48.1 & 25.6 \\
Otros & 22.8 & .2 & 22.6 & 99.3 \\
TOTAL & 808.7 & 401.2 & 407.5 & 50.4 \\
\hline
\end{tabular}

- Excluye servicio doméstico

Fuente: MIPLAN, 1990a, Cuadro E17

En el sector moderno, un gran número de asalariados permanentes es empleado por un pequeño número de propietarios de establecimientos grandes (Cuadro 2). La estructura ocupacional del sector informal es más heterogénea, e incluye propietarios de pequenos talleres, trabajadores familiares no remunerados y un gran número de trabajadores por cuenta propia y asalariados temporales. 


\section{Cuadro 2}

Distrlbución del empleo: Categorla ocupacional (Miles de trabaiadores)*

\begin{tabular}{|l|r|c|c|c|}
\hline Categorla & Total & Moderno & Informal & \% Informal \\
\hline Patrono & 31.5 & 7.8 & 23.7 & 75.3 \\
Cuenta propia & 206.3 & 4.3 & 202.1 & 97.9 \\
Familiar & 39.5 & 5.8 & 33.6 & 85.1 \\
Asalariado Perm. & 319.2 & 278.9 & 40.4 & 12.6 \\
Asalariado Temp. & 143.3 & 76.2 & 67.2 & 46.9 \\
TOTAL & 739.9 & 372.9 & 366.9 & 49.7 \\
\hline
\end{tabular}

- Excluye empleados del servicio doméstico

Fuente: MIPLAN 1990a, Cuadro E14

* La situación del sector informal se vuelve más interesante cuando se toman en cuenta los ingresos. Los trabajadores en el sector informal trabajan más horas que sus contrapartes en el sector moderno; mientras el $38 \%$ de los empleados en el sector moderno trabajaron más de 45 horas en la semana en referencia, $63 \%$ de los informales trabajaron más de 45 horas $^{15}$. No obstante, el ingreso mensual medio es menor en el sector informal.

\section{Cuadro 3}

Ingresos salarlales medlos por mes (colones):

Sector y rama de actlvidad, 1989.

\begin{tabular}{|l|r|r|r|c|c|l|}
\hline & \multicolumn{2}{|c|}{ GLOBAL } & \multicolumn{2}{c|}{ MODERNO } & \multicolumn{2}{c|}{ INFORMAL } \\
\hline Rama & Medla & Moda & Medla & Moda & Medla & Moda \\
\hline Agricultura & 376 & 248 & 425 & 281 & 250 & 205 \\
Mineria & 490 & 490 & 490 & 490 & - & - \\
Industria & 713 & 531 & 789 & 536 & 413 & 371 \\
Elect/Gas/Agua & 1119 & 916 & 1152 & 916 & 565 & 201 \\
Construcción & 617 & 386 & 718 & 527 & 509 & 359 \\
Comercio & 675 & 527 & 852 & 529 & 357 & 156 \\
Transp/Comun. & 768 & 601 & 951 & 763 & 602 & 593 \\
Financiero & 1231 & 691 & 1268 & 703 & 599 & 591 \\
Servicios & 705 & 151 & 937 & 899 & 275 & 155 \\
\hline TOTAL & 685 & 208 & 838 & 548 & 363 & 187 \\
\hline
\end{tabular}

Fuente: MIPLAN 1990a, Cuadro F17. 
Los menores ingresos en el sector informal no se explican por su concentración en actividades menos productivas. Las diferencias en los ingresos persisten aún cuando se compara a trabajadores de las misma actividad (Cuadro 3). Las diferencias también se presentan cuando se compara a trabajadores con la misma educación (Cuadro 4).

\section{Cuadro 4}

Ingresos salarlales medios por mes (colones): Sector y anos de estudio.

\begin{tabular}{|l|r|r|r|r|r|r|}
\hline & \multicolumn{2}{|c}{ GLOBAL } & \multicolumn{2}{c|}{ MODERNO } & \multicolumn{2}{c|}{ INFORMAL } \\
\hline Rama & Medla & Moda & Medla & Moda & Medla & Moda \\
\hline Ninguno & 343 & 209 & 430 & 268 & 273 & 182 \\
Menos de 4 & 434 & 211 & 554 & 289 & 319 & 175 \\
De 4 a 6 & 533 & 204 & 646 & 585 & 370 & 192 \\
De 7 a 9 & 614 & 574 & 713 & 548 & 401 & 203 \\
De 10 a 12 & 899 & 900 & 955 & 897 & 553 & 556 \\
13 0 más & 1215 & 924 & 1234 & 924 & 624 & 121 \\
\hline
\end{tabular}

Fuente: MIPLAN 1990a, Cuadro F19.

Asi, los trabajadores del sector informal ganan menos que trabajadores comparables en el sector moderno. La diferencia no puede atribuirse a diferencias en capital humano - al menos medido por la educación- ni a diferenciales de salarios entre clasificaciones globales de actividades. La situación del sector informal parece encajar con el marco de PREALC: se trata de un sector de pequenas empresas que usan pocos recursos complementarios al trabajo, y que opera en los márgenes competitivos fácilmente accesibles de cualquier mercado.

\section{C. ¿Qulén podría migrar?}

El primer paso en la argumentación general de este trabajo es establecer la existencia de un grupo de trabajadores que estarian dispuestos a migrar temporalmente entre el sector informal urbano y la recolección de cosechas agrícolas. Se espera haber presentado plausiblemente al sector urbano informal como un grupo grande de trabajadores que perciben bajos ingresos trabajando con poco capital en pequenas empresas en mercados de fácil entrada. Para descubrir las razones de la migración puede ser útil centrarse con mayor precisión en to inadecuado de los ingresos y la inestabilidad del empleo en el sector informal. 
La encuesta de 1989 encontró 202,058 empleados por cuenta propia en el sector urbano informal de El Salvador. Su ingreso modal mensual de todas fuentes era de 284 colones $^{16}$. El sector informal tenía 67,180 asalariados temporales, cuyo ingreso medio mensual era de 378 colones, y cuyo ingreso modal de todas las fuentes era de 271 colones ${ }^{17}$. Asimismo, había 50,625 trabajadores domésticos, con un ingreso mensual 192 colones y un ingreso modal de todas las fuentes de 196 colones $^{18}$.

Estos datos sobre ingreso nominal pueden traducirse a poder de compra. En febrero de 1990, el costo de una canasta de alimentación básica mínimamente adecuada se estimaba en 4.77 colones por persona diariamente ${ }^{19}$. Siguiendo la práctica del Banco Mundial, la línea de pobreza se establecia en dos veces el costo del mínimo nutricional, es decir, 9.54 colones por persona diariamente, o 286 colones por persona al mes. Así, trabajadores con ingresos como los descritos arriba apenas ganaban lo suficiente para alimentarse y vestirse a si mismos, y ya no se diga para cubrir gastos de vivienda. La encuesta de 1989 encontró que el hogar promedio está formado por 4.54 personas, y no es sorprendente que muchos hogares cuyos jefes son trabajadores del sector informal vivan en situación de pobreza, aún cuando más de un miembro de la familia trabaje ${ }^{20}$. De hecho, hay un estimado de 114,538 trabajadores del sector informal que son jefes de familias pobres ${ }^{21}$.

Aunque la necesidad puede obligar a los pobres a continuar trabajando, no hay razón para esperar que se mantendrán en un trabajo dado por mucho tiempo. Los patronos pueden despedir y reemplazar trabajadores con facilidad, y los trabajadores del sector informal podrían razonablemente dedicarse a la búsqueda de trabajo. Tienen poco que perder. Mientras $70 \%$ de los que trabajan en el sector moderno están cubiertos por la seguridad social o algún tipo de pensión, sólo el $3 \%$ de los trabajadores del sector informal tienen esa cobertura22. Mientras los propietarios de pequenos establecimientos comerciales podrian perder su lugar en el mercado al cambiar de trabajo o local ${ }^{23}$, no hay razón para esperar que los trabajadores temporales tengan la misma preocupación. Además, habiendo dejado un empleo informal, no será difícil para ellos encontrar otro en esta parte del mercado de trabajo de fácil entrada.

Datos sobre desempleo tienden a confirmar la hipótesis de cambios frecuentes de trabajo. La encuesta de hogares encontró 49,367 desempleados que habian trabajado antes, buscando activamente trabajo. EI empleo anterior de casi más de la mitad de ellos $(27,573)$ había durado menos de un ano24. 
Entre los desempleados que habian trabajado antes y estaban buscando trabajo la semana en referencia, $70 \%$ hablan estado buscando trabajo por menos de cuatro meses ${ }^{25}$. Entre los que habian sido asalariados temporales, $57 \%$ habian estado buscando trabajo por menos de dos meses. De los que habian trabajado como domésticos, 40\% habia estado buscando trabajo por más de dos meses; mientras que entre los empleados por cuenta propia, $75 \%$ habia buscado trabajo por menos de dos meses ${ }^{26}$.

Asi, San Salvador tiene un ejército no muy vasto de desempleados. Sin embargo, dentro del sector informal urbano, miles de trabajadores tienen empleos inestables con salarios sumamente bajos. Estos trabajadores pueden muy bien trasladarse temporalmente a labores agricolas. Luego de la recolección, siempre podrian regresar para autogenerarse empleo en el sector informal urbano de fácil entrada.

\section{Los trabajadores urbanos en la agrlcultura}

Las actividades de subsistencia fuera de la época de recolección de cosechas de los trabajadores agricolas con frecuencia tiene lugar en las ciudades. En 1980, más de un tercio de los trabajadores agrícolas de Sao Paulo vivian en áreas urbanas, mientras que en México un cuarto de los trabajadores agricolas eran de origen urbano ${ }^{27}$. En Guatemala, tres anos más tarde, 100,000 residentes urbanos trabajaban en la agricultura ${ }^{28}$. Al final de la década, 85,000 salvadorenos urbanos afirmaban que la agricultura era su actividad principal (Véase cuadro 1. arriba).

Hay dos desarrollos tras estos datos. El patrón de movilización de trabajo agricola está cambiando a medida que las relaciones salariales puras vencen al viejo patrón de dualismo funcional. Ese cambio, por su parte, refleja cambios en la producción, que tienden a incrementar la variación estacional de demanda de trabajo. Estos desarrollos aumentan significativamente la probabilidad de que los trabajadores se desplacen estacionalmente entre actividades rurales y urbanas.

\section{A. Más allá del duallsmo funcional}

Históricamente, los grandes terratenientes en América Latina han controlado el acceso a la tierra como una forma de asegurarse una oferta estacional de trabajo. Cuando el régimen de peones estricto perdió importancia, emergieron el dualismo funcional y el trabajo semiproletario $^{2 .}$. A los trabajadores se les permitió el acceso a pequenas parcelas de tierra para que se proveyeran de una parte de su subsistencia, pero las parcelas eran demasiado pequenas para proporclonar 
una cosecha adecuada. Asi, los pequenos agricultores fueron obligados a vender su trabajo por un salario en el mercado estacional.

\section{Cuadro 5}

Fuentes de Ingreso de pequenos agricultores.

\begin{tabular}{|l|c|c|c|c|c|}
\cline { 4 - 6 } \multicolumn{2}{c}{} & \multicolumn{3}{c|}{ Partic. en el Ingreso (\%) } \\
\hline & Ano & $\begin{array}{c}\text { Tamano } \\
\text { (ha) }\end{array}$ & Parcela & Salarlos & Otros \\
\hline El Salvador & 1975 & $0-1$ & 59 & 31 & 10 \\
Guatemala (N-E) & 1978 & $0-3.5$ & 29 & 59 & 12 \\
México (Puebla) & 1970 & $0-4$ & 32 & 58 & 11 \\
Colombia (G.Rovira & 1972 & $0-4$ & 79 & 16 & 5 \\
Ecuador (Costa) & 1974 & $0-5$ & 48 & 41 & 11 \\
Ecuador (Sierra) & 1974 & $0-5$ & 37 & 44 & 19 \\
\hline
\end{tabular}

Fuente: de Janvry, et.al., 1989b: p. 1212

Encuestas realizadas durante los setenta indicaban la dependencia de los pequenos agricultores de ingresos salariales obtenidos fuera de sus parcelas (Cuadro 5). Aunque el grado de inserción en relaciones salariales variaba de un lugar a otro, las familias con parcelas obtenian una parte significativa de su ingreso de la venta de su fuerza de trabajo.

Asi, al menos en alguna medida, los minifundistas constituyen la reserva de fuerza de trabajo para explotaciones comerciales. La historia, sin embargo, no termina alli. Para empezar, ignora el papel de los trabajadores rurales sin tierra. Aqui la evidencia en El Salvador es especialmente fuerte. Klein encontró que el número de familias rurales sin tierra en El Salvador aumentó de 30,541 (4.8\% de las familias rurales) en 1961 a $112,108(29.2 \%)$ en $1971^{30}$. Para 1975 , la cifra habla subido a $166,922,{ }^{2}$ casi el $41 \%$ de todas las familias rurales ${ }^{32}$.

La historia también no explica aumentos significativos en el empleo agrícola de residentes urbanos. En Sao Paulo, la participación de la población activa agrícola que era de origen urbano aumentó de $27 \%$ a $38 \%$ entre 1970 y 1980 . En México, subió de $23.8 \%$ a $26 \%$ durante el mismo periodo; mientras que en Perú se incrementó de $18.3 \%$ a $23.7 \%$ entre 1961 y $1972^{33}$. En la medida en que las periferias de las ciudades todavia son clasificadas como rurales en la estructura de algunos 
censos, estas estadisticas subestiman la importancia de los trabajadores urbanos.

A medida que la agricultura latinoamericana se desarrolla, sus "nuevos trabajadores temporales son en gran medida trabajadores puramente asalariados y no minifundistas como en el pasado ${ }^{\text {m4 }}$. El funcionalismo dual semiproletario se desintegra ${ }^{36}$, y se desintegra más rápidamente donde los trabajadores urbanos pueden ser empleados por las grandes propiedades.

"Aunque la estructura económica de los hogares campesinos, en los que el trabajo familiar genera ingreso de la parcela que poseen, les permite competir por niveles de salarios más bajos que una fuerza de trabajo completamente proletarizada (la teoría del dualismo funcional), el conflicto entre sus propias necesidades de trabajo y las necesidades de sus patronos en épocas de elevado empleo estacional operará en contra de los campesinos. Los trabajadores urbanos (una vez disponibles en abundancia debido a la expropiación de los campesinos a través del cambio en la legislación laboral y un reducido acceso a la tierra) son, en contraste, más fácilmente accesibles, y la concentración de asentamientos urbanos (urban dwellings) facilita y abarata a los contratantes el acceso a los trabajadores".

De esta manera, los trabajadores urbanos de subsistencia tienen en realidad una ventaja sobre sus contrapartes rurales en el nuevo mercado de trabajo agricola, especialmente cuando existe vivienda en el mismo lugar de trabajo para los trabajadores migrantes, cuando éstos pueden ser alojados por sus parientes rurales o cuando es posible el transporte diario desde la ciudad.

En El Salvador, estos desarrollos han significado el declive del colonato, el cual "habia desaparecido efectivamente como sistema importante de tenencia de la tierra para 1971".37 Una encuesta longitudinal encontró que mientras el $12.6 \%$ de las familias rurales todavla ocupaban casas como colonos en 1978 , sólo 5,5\% lo haclan en 1987 s. $^{\circ}$. Evidencia adicional sobre la extensión de las relaciones salariales es el hecho de que mientras el área cultivada cayó $23 \%$ durante esos anos de crisis, los días de trabajo contratado cayeron solo $2 \%{ }^{30}$.

Los trabajadores agrícolas, por lo tanto, son movilizados de manera creciente no sólo de entre los pequenos agricultores con parcelas subfamiliares y de entre los trabajadores rurales sin tierra, sino también de las ciudades. La combinación de residencia urbana y trabajo agrlcola en El Salvador es conocida, aunque no ha sido documentada. Tamo los trabajos de Deere y Diskirro como el de Klein ${ }^{41}$ hacen alusión a la 
migración urbano-nural para la época de recolección de cosechas en EI Salvador. Desafortunadamente, no existen datos precisos sobre la magnitud de este movimiento.

\section{B. Estaclonalldad y trabajo agricola}

La demanda de trabajo agricola siempre ha fluctuado con el cambio de estaciones. Ahora, sin embargo, cambios tecnológicos e institucionales parecen estar intensificando la variación estacional en la demanda de trabajo.

La estacionalidad agrícola está moldeada por la selección de la tecnología. En gran parte de América Latina, se ha aplicado la mecanización al cultivo pero no a la recolección. Esta mecanización parcial reduce la demanda de trabajo durante la época de crecimiento de los cultivos, mientras que la mantiene 0 inclusive la incrementa durante la recolección ${ }^{42}$. "La mecanización tiende a incrementar la estacionalidad del empleo excepto en las áreas más avanzadas, donde la mecanización de todas las etapas del proceso de trabajo ha sido completa'ws.

La estacionalidad también aumenta en la medida en que la agricultura pierde su diversidad. El cambio después de 1950 hacia el culttvo en gran escala de productos de exportación ha reducido la diversidad de los cultivos, reforzando asi las fluctuaciones estacionales en la demanda de trabajo ${ }^{44}$.

De acuerdo con un documento de trabajo preparado por PREALC justo antes de la crisis de los ochenta, en EI Salvador la estacionalidad del empleo rural constituia el obstáculo principal a un uso más adecuado del trabajo en la economia en generał5. Los meses de noviembre y diciembre son los de mayor actividad en la cosecha de café en el Salvador. Esa es también una época de cosecha de granos básicos. "Aproximadamente $85 \%$ del maíz y $80 \%$ del frijol se cultiva entre octubre y diciembre, $90 \%$ del sorgo de noviembre a enero y $80 \%$ del arroz entre septlembre y noviembre ${ }^{\text {net }}$. De esta manera, el mercado de trabajo para todos estos cultivos se vuelve más apretado para la misma época del ano.

Existen datos ilustrativos impresionantes en el conso agrlcola realizado en 1971, un ano que puede representar patrones agrícolas normales antes de los trastornos causados por la guerra. Los datos revelan marcados picos y fondos en el empleo agrícola (Cuadro 6). La demanda en diciembre era cinco veces mayor que tres meses antes. Mike Wise, de AID San Salvador, ha senalado que estos datos no captan el impacto del declive de la producción de algodón durante los 
ochenta. La producción de algodón en El Salvador cayó en $74.6 \%$ entre 1980-81 y 1988-89, mientras que el área cultivada disminuyó en $78 \%{ }^{47}$. Se cree que la caída en la producción de algodón disminuyó la demanda de trabajo agricola en los meses de abril a junio ${ }^{48}$. No obstante, la variación de diciembre a marzo es notable.

\section{Cuadro 6}

Empleo agricola mensual estimado (Equlvalentes a tlempo completo)

\begin{tabular}{|l|c|}
\hline Meses & Trabajadores \\
\hline Enero & 331,500 \\
Febrero & 332,400 \\
Marzo & 123,300 \\
Abril & 438,900 \\
Mayo & 442,700 \\
Junio & 454,500 \\
Julio & 401,000 \\
Agosto & 410,000 \\
Septiembre & 301,700 \\
Octubre & 204,300 \\
Noviembre & 691,300 \\
Diciembre & 672,300 \\
MEDIA & 400,300 \\
\hline
\end{tabular}

Fuente: Calculado de PREALC, 1979, P. 20

Además, en El Salvador, como en cualquier otro lugar, el grado de estacionalidad parece haber estado aumentando en lugar de disminuir. En 1961, el $31 \%$ de los requerimientos de trabajo agrícola anual se concentraba en el periodo noviembre-febrero. En 1971, el $42 \%$ del total del trabajo utilizado fue requerido durante ese periodo ${ }^{49}$.

La estacionalidad del empleo siguió aumentando durante los ochenta. Un estudio de 1987 reencuestó a un grupo de hogares fuera del área metropolitana de San Salvador, que habia sido encuestado en 1978. El estudio arroja "declives apreciables en la disponibilidad de durante todo el ano"so. La proporción de trabajadores involucrados durante el año en una sola actividad cayó de $81.9 \%$ en 1978 a $66.5 \%$ en $1987^{51}$. Se produjo una reducción de $10 \%$ en el número de traba- 
jadores por cuenta propia emepleados en la agricultura durante todo el anos.

Dado que la oferta de trabajo no es infinitamente elástica, las variaciones estacionales en la demanda de trabajo provocan variaciones estacionales significativas en el salario agricola. El documento de 1979 de PREALC, del cual se obtuvieron los datos del cuadro 6 , también afirma: "Los trabajadores agrícolas, incluyendo a las mujeres, pueden ganar 20 colones diarios durante la recolección de café en noviembre y diciembre, pero incluso los hombres ganan sólo de 2 a 3 colones diarios durante los meses inactivos"53.

En los últimos años los salarios rurales no han sido tan bien registrados como los ingresos en la ciudad, pero es claro que continúan variando ampliamente durante el ano agricola. De mayo de 1988 a abril de 1990, el salario mínimo agricola para adultos era de 10 colones diarios $^{54}$. En abril de 1990 aumentó a 11.5 colones. Para el ano cosecha 1989/90, el Ministerio de Agricultura estimaba que el salario diario en el cultivo de café era de 13 colones, mientras que el salario correspondiente para la recolección del grano era de más de 20 colones $^{55}$. De hecho, la recolección de café suele pagarse por obra. Personas familiarizadas con la cosecha $89 / 90$ estimaban que un trabajador calificado, sea rural o urbano, que había recolectado café antes, podia recolectar 10 arrobas al dia. Al salario prevaleciente de 5 colones por arroba, ésto implicaba un ingreso diario de 50 colones. Así, pereciera que los salarios de recolección pueden ser hasta 5 veces superior a los pagados fuera de la época estacional.

Resumiendo, la experiencia salvadorena parece ser consistente con los cambios en los mercados rurales de trabajo analizada por otros estudiosos de las economias latinoamericanas. La variación estacional en la demanda de trabajo es notable y creciente a medida que la mecanización se extiende a una parte del proceso de trabajo y a medida que las explotaciones agricolas se especializan en un número reducido de cultivos. La estacionalidad creciente, junto con los cambios institucionales, ha desplazado la demanda de trabajo de trabajadores permanentes hacia trabajadores temporales. Los trabajadores temporales provienen no sólo de pequenos agricultores con parcelas subfamiliares ni sólo de campesinos sin tierra. Los trabajadores agrícolas provienen también de la ciudad, donde las condiciones de contratación son táciles y donde no hay conflicto estacional en la demanda de trabajo en las parcelas de los trabajadores. 


\section{Todo tlene su tlempo}

Hace veinte anos, Harris y Todaro construyeron un modelo sumamente elástico para explicar la migración nural-ubano en el marco de altos niveles de desempleo urbanose. En el sistema Harris-Todaro, la migración rural-urbano tiene lugar "hasta que el ingresos real urbano esperado excede en el margen al producto marginal agricola"s7.

Un modelo en la tradición Harris-Todaro puede arrojar luz sobre las decisiones de los tabajadores urbanos ${ }^{50}$. El sector urbano formal debería incluirse dentro del rango de opciones de empleo. En realidad, parece que lo mejor es reemplazar la posibilidad del desempleo urbano por "empleo autogenerado" de PREALC en el sector informal. EI marco de decisión debería de permitir repetidas revisiones en cuanto a la selección del lugar de trabajo. Resulta crucial asumir que tanto el trabajo de recolección como el sector urbano informal son, de hecho, de fácil entrada, y que después de la recolección los migrantes podrán encontrar rápidamente un empleo en el sector informal. Como se sostuvo arriba, éste es un supuesto plausible.

Considérese un modelo de dos sectores, nural y ubano, definidos por el lugar de residencia. Los residentes rurales tienen como única opción el trabajo agricola. Asúmase que los residentes urbanos, aunque generalmente incorporados al trabajo urbano moderno o informal, también pueden aceptar trabajo agricola.

La actividad agricola paga un salario diario $w_{a} y$ ofrece mensualmente $n_{\mathrm{e}}$ dias de empleo por trabajador en el mes $t$. Las actividades informales urbanas pagan un salario $w_{n}$ y ofrecen mensualmente $n_{h}$ dias de trabajo. El sector moderno, por su parte, ofrece un salario $w_{m}$ el cual es tijado institucionalmente y no varía para equilibrar el mercado de trabajo. El empleo en este sector es bajo contratos que estipulan $n_{m}$ dias de trabajo al mes.

Los trabajadores que maximizan su ingreso aspiran a los trabajos que pagan salarios altos en el sector moderno. Asúmase que la actividad diaria en el sector informal urbano es una condición para estar en el lugar correcto y en el momento correcto para recibir una oferta de trabajo del sector moderno. Es decir, al trabajar fuera de la ciudad en labores agricolas, la probabilidad de recibir una oferta de trabajo del sector modemo es cero; mientras que si se trabaja en el sector informal se tiene una probabilidad positiva $p$ de incorporarse al sector moderno.

Ignorando la distinción entre los ingresos del trabajador medio y el 
marginal, el ingreso esperado mensual del empleo agricola es simplemente:

$$
E\left(y_{\mathrm{at}}\right)=E\left(w_{\mathrm{at}}\right)^{*} E\left(n_{\mathrm{at}}\right)
$$

Por otra parte, el ingreso mensual esperado de la actividad urbana informal incluye no sólo el rendimiento esperado por el trabajo informal mismo, sino el rendimiento posible en términos de una oferta de un trabajo (preferido) en el sector moderno. Suponiendo que las condiciones laborales en el sector modemo son establecidas institucionalmente, $w_{m}$ y $n_{m}$ se conocen con certeza. Asi:

$$
E\left(y_{h}\right)=p^{*} w_{m}^{*} n_{m}+(1-p)^{*} E\left(w_{h}\right)^{*} E\left(n_{h}\right)
$$

Estas dos ecuaciones simulan las opciones enfrentadas por un trabajador que se encuentra en actividades informales urbanas. Tal trabajador se desplazará hacia actividades agricolas si y sólo si:

$$
E\left(w_{a t}{ }^{*} E\left(n_{a 1}\right)>p^{*} w_{m t} n_{m t}+(1-p)^{*} E\left(w_{n}\right)^{*} E\left(n_{n}\right)\right.
$$

¿Es probable que ésto ocurra? La plausibilidad de la migración puede ilustrarse con la experiencia salvadorena. Para datos del área urbana, hay que volver nuevamente a la distribución del ingreso que aparece en la encuesta de hogares de $1989^{\circ 0}$. Para los salarios esperados en el sector moderno podría usarse el ingreso mensual modal de los asalariados permanentes del sector moderno, que es 897 colones $^{61}$. Se prefiere la moda sobre la media porque la distribución es altamente sesgada. Para la probabilidad de encontrar un trabajo permanente en el sector moderno puede usarse $0.25^{62}$.

¿Que opciones tienen los trabajadores en diciembre? Considérese la situación de un asalariado temporal en el sector informal. El ingreso mensual modal de estos trabajadores era de 206 colones $^{\text {es }}$. Usando esas cifras para los cálculos de ingreso esperado en la ciudad, estos trabajadores compararian los salarios agricolas esperados con el ingreso urbano esperado de 378 colones $^{\text {es }}$. Supóngase que a un trabajador se le ofrece sólo 14 dias de trabajo al mes recolectando café. Este trabajador se desplazará hacia el empleo agrícola durante la época de recolección si el salario diario de recolección es al menos 27 colones diarios ${ }^{65}$. Arriba se sostuvo que un trabajador en un buen cafetal puede ganar 50 colones diarios. Asi, es bastante plausible que el trabajador migraria. Si alguien esperara un menor salario esperaria también más dias de trabajo66. 
¿Cuáles serían las opciones en marzo, cuando el empleo agrícola es aproximadamente un cuarto del de diciembre (Cuadro 6)? Volvamos al caso del asalariado temporal. Supóngase que el salario agrícola diario cae al mínimo legal, en 1989, de 10 colones, pero no más abajo. Si al trabajador se le ofrecen las mismas dos semanas de empleo como en diciembre, éste seguramente regresaría a la ciudad. Aún si tuviera empleo todo el mes, los ingresos totales no superarian los 300 colones. Aunque esta cifra es mayor que ingreso mensual modal de los asalariados temporales del sector informal, está abajo de los 378 colones, que es el ingreso urbano esperado cuando se toma en cuenta la posibilidad de obtener un empleo en el sector formal.

Peter Gregory ha senalado que este modelo hace caso omiso de cualesquiera costos extras de manutención que los recolectores agricolas que viven fuera de sus hogares tienen. Sin embargo, esta omisión es razonable. Algunos contratantes proveen alimentación y vivienda, además del salario citado aquí. Algunos trabajadores viven con parientes que se han quedado en las zonas rurales del país, aumentando sus costos de manutención muy poco con relación a los que tendrian en la ciudad.

Esta condición de equilibrio al estilo Harris-Todaro demuestra bastante simplemente la plausibilidad de la migración estacional entre el sector unano informal y las labores de recolección. Desafortunadamente, no revela efectos de retroalimentación entre las variables de un período a otro. Tampoco revela efectos acumulativos importantes en la medida en que las decisiones de distintos trabajadores son agregadas.

Estos efectos acumulativos pueden operar a través de la demanda. Una persona contrata a un trabajador por cuenta propia para que le repare una gotera, el carpintero compra el almuerzo en el comedor que está al lado y el dueño del comedor, por su parte, compra una olla al vendedor que se encuentra en la esquina de la calle. Qué es lo que inicia el ciclo. Comenzó posiblemente con los ingresos que la primera persona obtuvo de la recolección de café, de manera que los ingresos agricolas estimularian la actividad urbana.

Los efectos pueden darse también a través de la oferta. Considérese de nuevo la situación en marzo. Varios cientos de miles de trabajadores no podrian dejar sus labores agricolas e incorporarse al sector urbano informal sin afectar los ingresos urbanos, sobre todo si se tiene en cuenta la facilidad de entrar a los mercados competitivos. Si nit no cae, wit probablemente lo hará, a la manera en que se sefiala en el modelo de PREALC. Así, es probable que la migración estacional entre 
las actividades urbanas y las agricolas introduzca variaciones estacionales significativas en los ingresos de los pobres urbanos.

Cuando se estaba preparando la revisión final de este trabajo, Juan Pablo Pérez Sainz nos comentó sobre sus estudios de caso de microempresas en Centro América. Estos estudios encontraron variabilidad estacional de los ingresos en el sector informal que nuestro argumento predice.

Resumiendo, las explotaciones agricolas parecen estar volcándose de manera creciente al reclutamiento de fuerza de trabajo urbana para abastecerse de trabajadores temporales durante la época de recolección. Los trabajadores del sector moderno no arriesgaria empleos permanentes altamente remunerados para aceptar trabajo agricola estacional. ¿Quienes aceptan la oferta de trabajos agricolas? Los contratantes dependen del sector urbano informal. Esta sección ha ilustrado la plausibilidad de la decisión de un trabajador informal para cambiarse temporalmente a la agricultura y luego regresar a actividades urbanas informales de fácil entrada, un patrón de empleo que seguramente debe introducir variaciones estacionales notables en los ingresos de los trabajadores del sector urbano informal.

Una advertencia final: el centrarse en las oportunidades de salarios y empleo hace caso omiso de otras consideraciones serias. En EI Salvador, puede optase por trabajo urbano en lugar de trabajo rural aún cuando existan oportunidades restringidas de ingresos si la ciudad provee un mejor acceso a servicios sociales y menos vulnerabilidad a la agresión militar.

\section{IV. "Pulgarclto" senala el camino}

Después del terremoto de 1986, los autobuses en El Salvador portaban calcomanias con una colorida senal del pulgar hacia arriba y la frase "Arriba pulgarcito". El pulgarcito, naturalmente, es EI Salvador mismo: alegre, astuto y pequeno, "un pequeho gran pais".

El pequeno tamano de El Salvador ("Pulgarcito") lo ha colocado entre los líderes de la transformación estructural de los mercados de trabajo que actualmente está teniendo lugar en América Latina. El trabajo asalariado agricola y el sector urbano informal están siendo integrado en un solo gran mercado. De manera creciente, variaciones en la demanda estacional de trabajo agricola son satisfechas mediante la contratación temporal de trabajadores de la periferia urbana y que trabajan en el sector urbano informal, introduciendo un grado mayor de 
estacionalidad en los ingresos de los pobres urbanos.

Mediante la identificación de nuevos canales de efectos de retroalimentación, la modificación de modelos del mercado laboral plantea nuevos interrogantes de política económica. Como ilustración, puede considerarse cualquier política dentro del conjunto de posibles políticas disenadas para aumentar las exportaciones agricolas: devaluación, aplicación de un sistema de tasas de cambio múltiples, disminución de aranceles sobre fertilizantes importados, tasas de interés preferenciales, establecimiento de acuerdos internacionales para respaldar el precio mundial de un producto de exportación, etc.

Supóngase que El Salvador decide promover las exportaciones de café. La demanda de trabajo agrícola aumentará en noviembre y diciembre. Esta demanda entra en conflicto con las necesidades estacionales de trabajo en las parcelas trabajadas por pequehos agricultores, de manera que se recurrirá a trabajadores temporales dentro los campesinos sin tierra y el sector informal urbano. El patrón de migración identificado en este trabajo se intensificará. El empleo en el sector urbano informal se volverá aún menos estable y los ingresos de los pobres urbanos fluctuarán todavia más.

Por su puesto, este es una análisis de equilibrio parcial, como to son también los análisis que enfatizan los efectos positivos del aumento en los ingresos de divisas. Lo que los analistas necesitan realmente es un modelo de equilibrio general de la economía salvadoreha, que pueda seguir la pista a los amplios efectos de cualquier cambio de política económica. En este trabajo se espera haber mostrado que un modelo de equilibrio general deberia permitir migración estacional entre el sector urbano informal y la agricultura.

\section{BIBLIOGRAFIA}

Briones, C. (1988), "Mercados de trabajo y subutilización de la fuerza de trabajo en El Salvador", Realidad Económico-Social, Año 1, No 5 (septiembreoctubre, 1988).

Briones, C. (1987), "Un ensayo de descripción de las caracterlsticas del sector informal urbano salvadoreño", Boletín de Ciencias Económicas y Sociales, Año X, No 4 (julio-agosto, 1987).

Deere, C.D. and M. Diskin (1983), Rural Poverty in El Salvador Dimensions, Trends, and Causes, mimeo prepared for the Rural Employment Policios Branch, International Labour Organization, june, 1983.

de Janvry, A. (1981), The Agrarian Question and Reformism in Latin Amerka, (Baltimore: Johns Hopkins, 1981). 
de Javry, A., and E. Sadoulet (1989b), "Investment Strategies to Combat Rural Poverty: A proposal for Latin America", World Development, Vol. 17, № 8 (August, 1989).

de Janvry, A., E. Sadoulet, and L. W. Young (1989), Land and Labour in Latin American Agriculture from the 1950s to the 1980s", The Journal of Peasant Studies, Vol. 16. N2 3. (April, 1989).

de Janvry, A., E. Sadoulet, and L. Wilcox (1989c), "Rural Labour in Latin America", International Labour Review, Vol. 128, N 6 (november-december, 1989).

Dervis, K., J. de Melo, and S. Robinson (1982), General Equilibrium Models for Development Policy (New York: Cambridge University Press, 1982).

FUSADES. Boletín Económico y Social, NN 40 (Diciembre, 1988), NN 46 (septiembre, 1989), y N2 47 (octubre, 1989).

Harris, J., and M. Todaro (1970), "Migration, Unemplyment, and Development: A Two-Sector Model", American Economic Review, Vol. 60, pp. 126-141.

Klein, E. (1985), "El impacto heterogéneo de la modernización agricola sobre el mercado de trabajo", Socialismo y participación, Vol. 30 (june, 1985).

Klein, E. (1980), "Pauperización campesina: empleo $\theta$ ingresos agrícola", Nueva Antropolgía, Año IV. No 13-14 (1980).

Menjivar Larín, R., and J. P. Sainz, eds. (1989), Informalidad en Centroamérica: Evidencias o interrogantes, (Guatemala: FLACSO y Fundación Friedrich Ebert, 1989).

Ministerio de Trabajo (1990), El Salvador, Ministerio de Trabajo y Previsión Social, Departamento de Planificación, "Estudio Socioeconómico para la Revisión y Determinación de Salarios Minimos en la Industria, Comercio, Servicios y Sector Agropecuario", mimeo, San Salvador, marzo, 1990.

MIPLAN (1990), EI Salvador, Ministerio de Planificación, Unidad de Investigaciones Muestrales, Encuesta de Hogares de Propósitos Múltiples, Total País Urbano, 1989; tabulaciones cruzadas sin publicar impresas on San Salvador on Marzo, 1990.

PREALC (1979), El Salvador: Proyecto de Generación de Empleos en el Corto Plazo, 2 volumenes, Documento de Trabajo 166, Enero, 1979.

PREALC (1977), Situación y Perspectivas del Empleo en El Salvador, 2 volumenes (Santiago: ILO, 1977).

Reinhardt, N. (1987), "Agro-Exports and the Peasantry in the Agrarian Reforms of El Salvador and Nicaragua," World Development, Vol. 15, No. 7 (July, 1987).

Taylor, L., ed. (1990), Socially Relevant Policy Analysis: Structuralist Computable General Equllibrium Models for the Developing World (Cambridge, MA: MIT Press, 1990).

Tokman, V. (1986), "Adjustment and Employment in Latin America: The current Challenges," International Labour Review, Vol. 125, No. 5 (September-October, 1986).

Tokman, V. (1987), "El Sector Informal: Quince Años Después," El Trimestro Económico, Vol. LIV, No. 3 (Julio-Septiembre, 1987). 
Tokman, V. (1988), "Urban Employment: Research and Policies in Latin America," CEPAL Review, 34.

Tokman, V. (1989), "Policies for a Heterogeneous Informal Sector in Latin America," World Development, Vol. 17. No. 7 (July, 1989).

United States. Senate. Committee on Foreign Relations, (1989), Foreing Assistance Authorization for Fiscal Year 1990, Hearings. Y4.F76/2:S.hrg. 101293.

Williams, R. (1986), Export Agriculture and the Crisis in Central America (Chapel Hill: University of North Carolina Press:1986).

\section{NOTAS}

1. Proceso 387, 7 de junio de 1989.

2. Gaes, 1990, pp. 117-119.

3. Gregory, 1986.

4. Mauricio Alens, Director de Coordinación del Ministerio de Planificación, facilitó al autor tabulados de los resultados de la Encuesta de Hogares de Octubre 1988-febrero 1989. Estos tabulados se citan on la bibliogralla COmo MIPLAN, 1990a.

5. PREALC, 1977, pp. 1 y 2. Los datos son de 1974.

6. Alan Wood, de AID, declaró ante el Congreso de los Estados Unidos el 2 de mayo de 1989: "Quisiera agregar algo más sobre Hernando de Soto, si puedo hacerlo. Probablemente, una de las mejores cosas que AID ha hecho fue darle $\$ 12,000$ hace algunos años para que iniciara su programa. Esos $\$ 12.000$ fueron bien gastados. El ha tenido un impacto sobre América Latina que es difícil de estimar". Senado de los Estados Unidos 1989, pp. 32-33.

7. de Soto, 1989.

8. Portes y Walton, 1981.

9. PREALC, 1977, p. 4.

10. PREALC, 1977, p. 13.

11. Tokman, 1988, p. 144.

12. PREALC, 1977, pp. 258-259.

13. MIPLAN, 1990b, vol. I. p. 36.

14. MIPLAN, 1990a, cuadros E23 y E55.

15. MIPLAN, 1990a, cuadro E25.

16. MILAN, 1990a, cuadros E23 y F25.

17. MIPLAN, 1990a, cuadros E23, F20 y F25.

18. MIPLAN, 1990a, cuadros E30, F20 y F25. Mike Wise de AID. El Salvador encuentra estas cifras sobre trabajadores domésticos implausiblemente bajas.

19. Ministerio de Trabajo, 1990, cuadro 4.

20. MIPLAN, 1990a, cuadro 0.

21. MIPLAN, 1990a, cuadro F12.

22. MIPLAN, 1990a, cuadro E30. 
23. Entrevista con Carlos Briones, San Salvador, mazo de 1991.

24. MIPLAN, 1990a, E68.

25. MIPLAN, 1990a, E72.

26. MIPLAN, 1990a, E77.

27. de Janvry et. al., 1989b, p. 1213.

28. Klein, 1985, p. 28.

29. de Janvry, 1981.

30. Klein, 1980, p. 113.

31. Klein, 1980, p. 113.

32. Deere y Diskin, 1983, p. 23.

33. de Janvry et. al., 1989b, p. 1213.

34. Klein, 1985, p. 28.

35. de Janvry et. al., 1989c, p. 724.

36. de Janvry, 1989c, pp. 715-716.

37. Deere y Diskin, 1983, p. 25.

38. Gore et. al., 1987, p. 33.

39. Ibid., p. 49.

40. Deer y Diskin, 1983, pp. 30 y 40.

41. Kelin, 1980, p. 115.

42. Klein, 1985, p. 25.

43. de Janvry, 1989c, p. 725.

44. Klein, 1985, p. 27.

45. PREALC, 1979, p. 13.

46. FUSADES, 1989, № 47, p. 4.

47. Calculado de MILAN, 1990c, pp. 43 y 44.

48. Entrevista con Mike Wise, San Salvador, marzo de 1991.

49. Deere y Diskin, 1983, p. 36.

50. Gore et. al., 1987, p. $x$.

51. Ibid., p. 60.

52. Ibid., p. 74.

53. PREALC, 1979, p. 17.

54. Ministerio de Trabajo 1990, cuadro 3.

55. Ministerio de Trabajo, 1990, cuadro 14.

56. Harris y Todaro, 1970.

57. Ibid., p. 127.

58. J.P. Pérez Sainz me llamó la atención sobre Mezzera, 1987, pp. 15-16, donde se discuten otras adaptaciones al modelo Harris-Todaro, entre ellas Fields, 1975.

59. Si los empleos formales son seguros en el tiempo, la ganancia obtenida de cambiarse al sector formal no es captada completamente por salario mensual. Podrla ser útil reformular el modelo en términos del valor presente descontado del flujo de ingresos esperados.

60. MIPLAN, 1990a.

61. Ibid., cuadro F20.

62. No está nada claro sobre cuál es la mejor forma de estimar p. Dado que se carece de datos sobre tasas de contratación y de rotación, se ha 
decidido simular el enfoque original Harris-Todario (p. 129). Estos autores fijan el salario urbano esperado igual a salario urbano medio, es decir, multiplicado por la probabilidad de encontrar un trabajo. Esta probabilidad se calculó dividiendo el número de empleados urbanos entre el de la población económicamente activa urbana (empleados o desempleados). La estadistica correspondiente en nuestro modelo serla el número de empleados en el sector moderno dividido entre el total de personas trabajando en el área urbana. Esto arroja un p de 0.5 (MIPLAN, 1990, cuadro E22). Sin embargo, para alguien que se desplaza hacia el mercado de trabajo urbano lo más probable es encontrar trabajo inicialmente en una de las tres categorlas ocupacionales: trabajador por cuenta propia, asalariado temporal o servicio doméstico. Dentro de estas categorlas, la proporción de empleos en el sector moderno, p, es de sólo 0.20 (lbid., cuadro E30).

63. MIPLAN, 1990a, cuadro F20.

64. $02.5 \times 897+0.75 \times 206$.

65. $(0.25 \times 987+0.75 \times 206) / 14^{\circ}$.

66. Se ha sugerido que los asalariados temporales pueden tender a ser jóvenes sin experiencia rural y con poca disposición a recolectar café. No se dispone de historias de empleo, pero MIPLAN, 1990a, muestra que el 49\% de los asalariados temporales tenlan una edad de 30 ah́os o más (Cuadro E31).

67. Dervis et. al., 1982; Taylor, 1990. 